\title{
OntoFire: an ontology-based geo-portal for wildfires
}

\author{
K. Kalabokidis, N. Athanasis, and M. Vaitis \\ University of the Aegean, Department of Geography, 81100, Mytilene, Greece \\ Received: 3 August 2011 - Revised: 3 October 2011 - Accepted: 6 October 2011 - Published: 5 December 2011
}

\begin{abstract}
With the proliferation of the geospatial technologies on the Internet, the role of geo-portals (i.e. gateways to Spatial Data Infrastructures) in the area of wildfires management emerges. However, keyword-based techniques often frustrate users when looking for data of interest in geo-portal environments, while little attention has been paid to shift from the conventional keyword-based to navigation-based mechanisms. The presented OntoFire system is an ontologybased geo-portal about wildfires. Through the proposed navigation mechanisms, the relationships between the data can be discovered, which would otherwise not be possible when using conventional querying techniques alone. End users can use the browsing interface to find resources of interest by using the navigation mechanisms provided. Data providers can use the publishing interface to submit new metadata, modify metadata or removing metadata in/from the catalogue. The proposed approach can improve the discovery of valuable information that is necessary to set priorities for disaster mitigation and prevention strategies. OntoFire aspires to be a focal point of integration and management of a very large amount of information, contributing in this way to the dissemination of knowledge and to the preparedness of the operational stakeholders.
\end{abstract}

\section{Introduction}

In an era of technology advances, society and the environment all over the world are still affected by the threat of wildfires. Mega-fires in southern European and Mediterranean forest ecosystems destroy thousands of hectares in countries like France, Greece, Italy, Portugal, Spain and elsewhere every year. In the Mediterranean basin, the number of ignitions

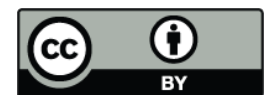

Correspondence to: K. Kalabokidis (kalabokidis@aegean.gr) and the area affected by fire increased exponentially over the last decades (Pausas et al., 2008). In the United States, the annual area burned by wildfire has increased in the last decade, despite large expenditures and infrastructures to fire suppression (Robichaud et al., 2007). In the summer of 2010 alone, widespread forest and peat wildfires in western Russia burned over hundreds of thousands of hectares and directly caused the death of more than 50 people (Stocks et al., 2011).

With the proliferation of the Internet technology, many agencies deliver their up-to-date wildfire-related content in the World Wide Web. The research trends in the geospatial domain show the shift from monolithic environments, where the involved agencies are responsible for producing, archiving and updating their data on their own, to distributed environments, where spatial data and resources are exchanged and shared over the web (Bernard et al., 2003). Open source solutions (e.g. MapServer, 2011; GeoServer, 2011) and commercial systems (e.g. ESRI, 2011), combined with programming interfaces like Google Maps (2011) and Microsoft Bing Maps (2011), provide the means for organizations to develop web-based applications specialized in wildfire management. These technologies allow monitoring and early warning systems to display their daily content about fire danger maps, maps of active fires and fire forecast, fire risk and soil moisture maps, satellite images, air quality images, etc. (Grasso, 2009).

The role of Spatial Data Infrastructures (SDI) for an effective decision-making has received extensive contribution from researchers in the area of natural disasters (Annoni et al., 2005; Köhler et al., 2006; Köhler and Wächter, 2006; Giuliani and Peduzzi, 2010), especially in the area of forest fires (Christensen et al., 2006; Galindo, 2010). Geoportals play the role of SDI gateways by providing single access points to multiple emergency data and facility resources. Geo-portals about wildfires can provide valuable help for user communities about risk knowledge, prevention strategies, management schemes and disseminating information.

Published by Copernicus Publications on behalf of the European Geosciences Union. 


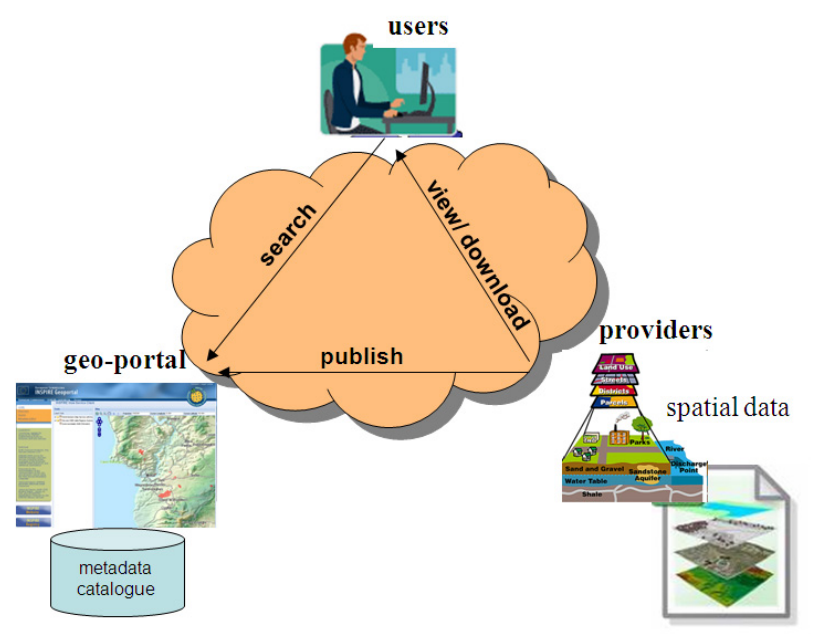

Fig. 1. Publishing, searching and using information in a geo-portal environment.

They act as "negotiators" between users and information providers (Fig. 1). Providers share their emergency spatial data by publishing corresponding declarative metadata, whilst the geo-portal receives the metadata published and organizes them in metadata catalogues. Users search for suitable information by querying the portal's metadata catalogues for relevant matches. Results that satisfy the users' needs can be viewed or downloaded from the providers' side. Figure 1 represents this inter-communication between users and providers in a geo-portal environment.

Nevertheless, searching for resources in geo-portals remains a frustrating task. There has been extensive work regarding the limitations of keyword-based techniques used for information retrieval in SDI, where users may not know which keywords to use, have too little information on how to fill in interactive forms and find it difficult to estimate how many filter criteria to be utilized (Hochmair, 2005; Klien et al., 2006; Lutz, 2006; Lutz and Klien, 2006, Shvaiko et al., 2010). The use of ontologies and the Semantic Web (Berners-Lee et al., 2001) is seen as a part of the solution to overcome limitations of the current search mechanisms. In the area of natural disasters, ontology-based solutions have been proposed to provide seamless integration of heterogeneous data agencies (Silva and Madurapperuma, 2007; Murgante et al., 2009) and efficient information retrieval (Babitski et al., 2011). There has not been much work, however, on the improvement of the information retrieval mechanisms from a user's perspective. Little attention has been paid to the shift from the conventional keyword-based to navigationbased mechanisms. There are situations where browsing through hyperlinked pages is more appropriate than querying, such as where users have no clear idea of their information need, or they are not proficient with the keyword-based techniques provided (Lucarella and Zanzi, 1996). By following navigation-based mechanisms, useful resources can be explored in a more intuitive way (Athanasis et al., 2005, 2009), and time is not wasted to scan through the results to reject those that are irrelevant (Marshall and Shipman, 1997).

In this work, we present OntoFire, an ontology-based geoportal about wildfires. A domain ontology for wildfires is exploited that semantically organizes the metadata provided, while users exploit the ontology during their navigation in the system. Instead of only typing keywords, the semantic relationships of the ontology's concepts are translated to hyperlinks that connect semantically related pieces of information. Through these hyperlinks, the system allows the navigation to different data sets which are semantically related, according to the wildfire ontology. Furthermore, the semantic navigation techniques are combined with analogous capabilities of spatial navigation. At every browsing action, users of OntoFire can discover resources based on their topological relationships, e.g. resources that exist within a geographical area of interest or in a zone buffered around this area. Every resource found is a potential starting point for further exploration by leveraging its semantic and spatial relationships with other resources. Through the semantic and spatial navigation-based mechanisms, the relationships (both semantic and spatial) between the data can be discovered, which would otherwise not be possible when using querying techniques alone. This harmonic coexistence of semantic and spatial navigation mechanisms is the most innovative feature of the proposed approach.

\section{Background}

There has been extensive research of ontologies in the area of natural disasters. Most of the approaches identify their role for seamless integration and management of heterogeneous, multi-lateral data from different agencies. Joshi et al. (2007) describe a Disaster Mitigation Modeling System that uses ontologies for tying together information from various fields of disaster mitigation. In Ilyas and Ahmed (2010), ontologies are exploited for the automatic data gathering, analysis and decision making in a disaster management system, while Zaharia et al. (2009) discuss how intelligent agents can extract knowledge to support local or government decisions in better handling the consequences of disasters. Most of these approaches, however, do not exploit the rich semantics of the underlying data to provide efficient ways for information retrieval. In Klien et al. (2006), the motivating scenario is taken from the domain of forest ecology: to retrieve data of interest, users specify their query terms in a query template based on a shared vocabulary. In a similar approach, a prototype system has been developed to support catalogue services for information related to disaster management (Lan et al., 2008). The proposed query interface for information discovery provides the opportunity to specify query criteria and constraints to refine the semantic queries. 
Gwenzie (2010) implies ontology visualization as a means to access resources of forecasting of severe weather events. By visualizing the hierarchy of concepts, the system provides visual aid of the underlying semantics. However, information retrieval is still based on keywords, and ontology visualization is only used as assistant tool for the query formulation. In Babitski et al. (2011), ontology-based visualization is accomplished through a Semantic Data Explorer (Paulheim and Meyer, 2011). Nevertheless, the proposed system is not a geo-portal environment but rather an integrated emergency management system for connecting existing systems and databases.

Research activities have been developed to examine the advantages of ontology exploitation in spatial query languages. Kolas (2008) and Zhai et al. (2010) adopt a solution to enhance the semantic query language SPARQL with spatial characteristics. Fu et al. (2005) present semantic query expansion techniques to support the retrieval of documents that are considered to be spatially relevant to user's queries. Even though the aforementioned approaches combine semantic query languages with spatial operators, they have not been applied to enhance the searching mechanisms in geo-portal environments.

All in all, there is still a considerable knowledge gap on how to find useful information in geo-portal environments by browsing using hyperlinks instead of keyword-based querying. This paper takes a first step in addressing this gap. We propose a wildfire ontology that serves as the conceptual backbone in OntoFire. Based on this ontology, end users are expected to exploit the proposed innovative semantic and spatial navigation mechanisms to find data of interest, while data providers may also afford the privilege to enrich the system with new semantically annotated metadata descriptions.

\section{The ontology of OntoFire}

The ontology of OntoFire includes several concepts in order to describe the semantic context of wildfires and their associated risks. Risks depend on both physical and socioeconomic factors (Blaikie et al., 1994; Cannon, 1994). Equation (1) represents the relationship between these concepts (Chen et al., 2003):

risk $=$ hazard $\times$ vulnerability

According to Pyne et al. (1996), hazards are the result of interaction between biophysical factors (i.e. physical geography), such as vegetative fuels, topography and weather. Wildfires are affected by the vegetative fuels because the quantity, size, density, quality, continuity and moisture content of the vegetation determine the availability of fuel for combustion (Vasilakos et al., 2009). Topography modifies the general climate of a region and thereby affects the availability of fuels and fire behavior. Furthermore, fire weather (i.e. wind, temperature, humidity and rainfall conditions) influence fire ignition and behavior (Schroeder and Buck, 1970). Vulnerability can be defined as the degree to which population, built environment and socioeconomic activities are prone to damage from a hazard event with physical aspects (Kalabokidis et al., 2002; Chen et al., 2003).

The terms "natural risk", "hazard" and "vulnerability" make up the backbone of the OntoFire ontology. In particular, relationship "hazard" expresses the semantic association between concept "Natural Risk" and concept "Physical Environment", because resources about natural risks are influenced by biophysical factors, i.e. resources about the physical environment. In a similar way, relationship "vulnerability" expresses the semantic association between concept "Natural Risk" with concept "Infrastructure", because information about natural risks are influenced by anthropogenic factors, i.e. resources of class "Infrastructure".

At the most upper level of the OntoFire ontology, the generic class "Geo-Information Resource" holds properties of the basic core of ISO 19115 metadata standard (Lee and Chan, 2000) (Fig. 2). ISO 19115 is the most commonly used metadata standard in geo-portals, compared with other metadata standards like the Content Standard for Digital Geospatial Metadata and the INSPIRE metadata standard (Drafting Team, 2009). Due to the extensive set of the ISO 19115 metadata (around 300 metadata elements), OntoFire only uses the basic core metadata element set of ISO 19115. Every element of the basic core set corresponds to an attribute of the generic class "Geo-Information Resource". As a consequence, every resource classified under any class of the OntoFire ontology can be described with metadata derived from the basic core set of ISO 19115. Figure 2 represents graphically the generic class "Geo-Information Resource" and its properties that correspond to the basic core elements of the ISO 19115 metadata standard.

Beyond the generic class "Geo-Information Resource", the terms "Natural Risk", "Infrastructure" and "Physical Environment" make up the basic classes in the OntoFire ontology, while "hazard" and "vulnerability" constitute relationships between them. Natural risks (i.e. resources classified under class "Natural Risk") can be further specialized into climatic and geological. Earthquakes are specialized categories of geological risks. In a similar way, climatic risks are specialized to atmospheric (e.g. storms), hydrological (e.g. floods and draughts) and biophysical (e.g. wildfires) natural risks. Class "Fire" is a sub-class of class "Biophysical".

Infrastructures are further categorized into urban areas, road networks, land uses, objects of type "Ownership \& Jurisdiction", fuel breaks, high danger areas (e.g. gas stations, landfills, power lines and army activities) and "Fire Management" objects (i.e. firefighting outposts and firefighting vehicles). Class "Physical Environment" is related with classes "Meteorology_and_Climate", "Topography" and "Vegetation" to express the affiliation of climate, topography and vegetation to the physical environment of a particular area. Subclasses of class "Meteorology_and_Climate" are 


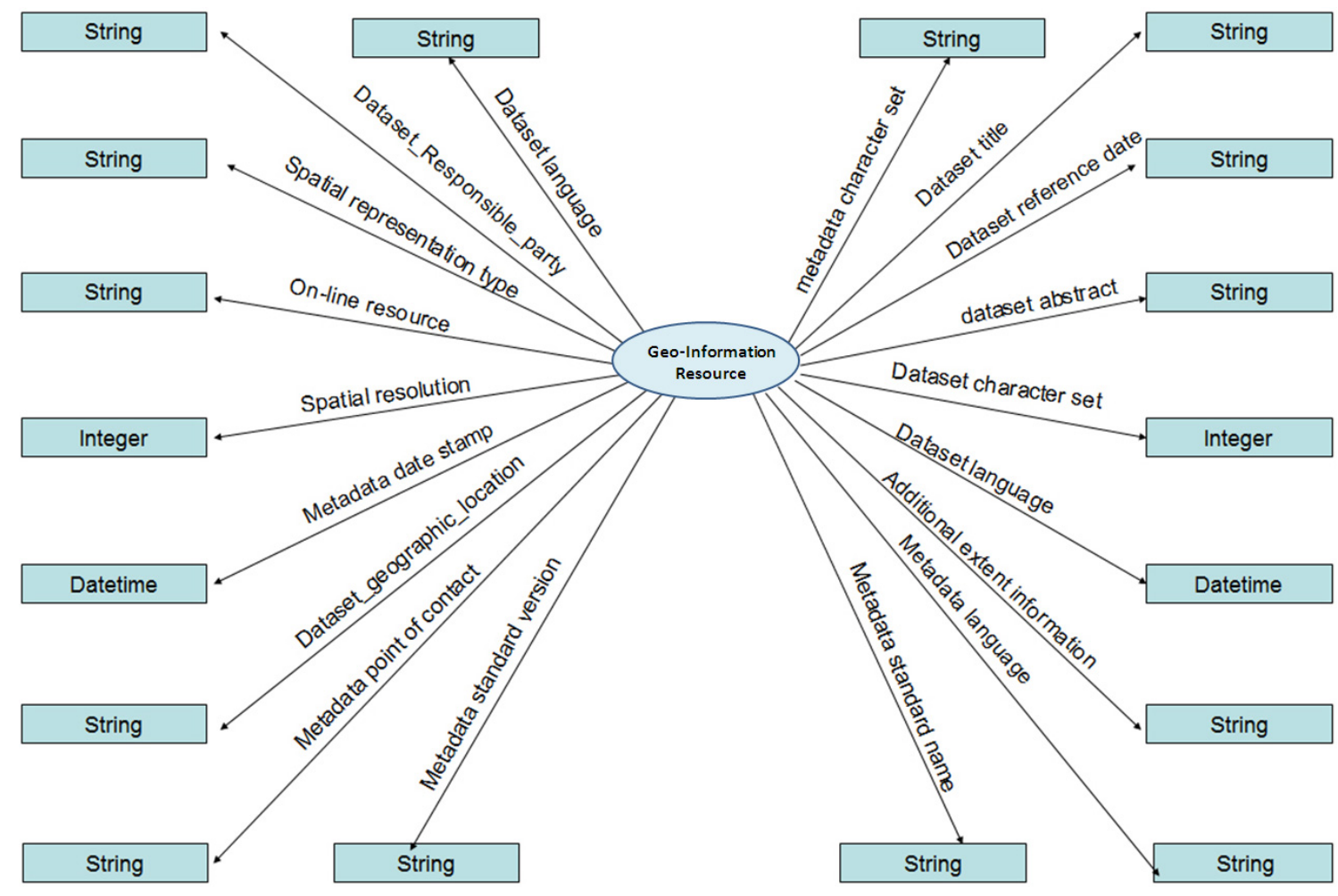

Fig. 2. The generic class "Geo-Information Resource" and its properties that correspond to the basic core elements of the ISO 19115 metadata standard.

"Weather Monitoring" and "Weather Prediction". Subclasses of class "Topography" are "Contour", "Coast line", "Hydrographic network" and "Elevation Model". Finally, class "Vegetation" is specialized to subclasses "Fuel type/Model" and "Cover type".

Figure 3 represents the OntoFire ontology. We apply the RDF Schema Definition Language (RDF Schema) (Brickley and Guha, 2004) to express the vocabulary of terms in the ontology. RDF Schema is an abstracted level of the Resource Description Framework (RDF) (Lassila and Swick, 2001), which in turn is a metadata standard used to represent metadata descriptions. In RDF Schema, classes represent abstract entities referring to sets of similar resources, while properties represent attributes (i.e. characteristics of a class) or relationships among classes. Classes and properties are represented as directed graphs. The arcs generally represent the properties, each of them connecting two nodes, coming from a class (drawn as an oval) and pointing to another class or a characteristic of a class (drawn as a rectangle). In OntoFire ontology, only the generic class "Geo-Information Resource" contains characteristics. These characteristics correspond to the basic core elements of the ISO 19115 metadata standard, as shown in Fig. 2.

\section{The OntoFire geo-portal}

OntoFire provides two key functionalities. First, end users can use the browsing interface to find resources of interest by using the semantic and spatial navigation mechanisms provided. Second, data providers can use the publishing interface to submit new metadata, modify metadata or remove metadata in/from the catalogue. The browsing interface is open to everyone, while the publishing interface can be used only by authorized users.

\subsection{The browsing interface}

Searching for useful resources is based on exploiting the semantic and spatial relationships of the geo-portal's resources. The flowchart of Fig. 4 shows the basic principles of OntoFire's navigation mechanisms. From a starting page, users can specify semantic and spatial criteria and find resources classified under a specific category (i.e. ontology class). By choosing a resource from the result list, users obtain the detailed description of the corresponding resource. Every resource is a potential starting point for further exploration and allows users to continue their navigation spatially or semantically. A resource that satisfies the user's needs can be viewed or downloaded from the provider's server. At every browsing step, users can define a different navigation 


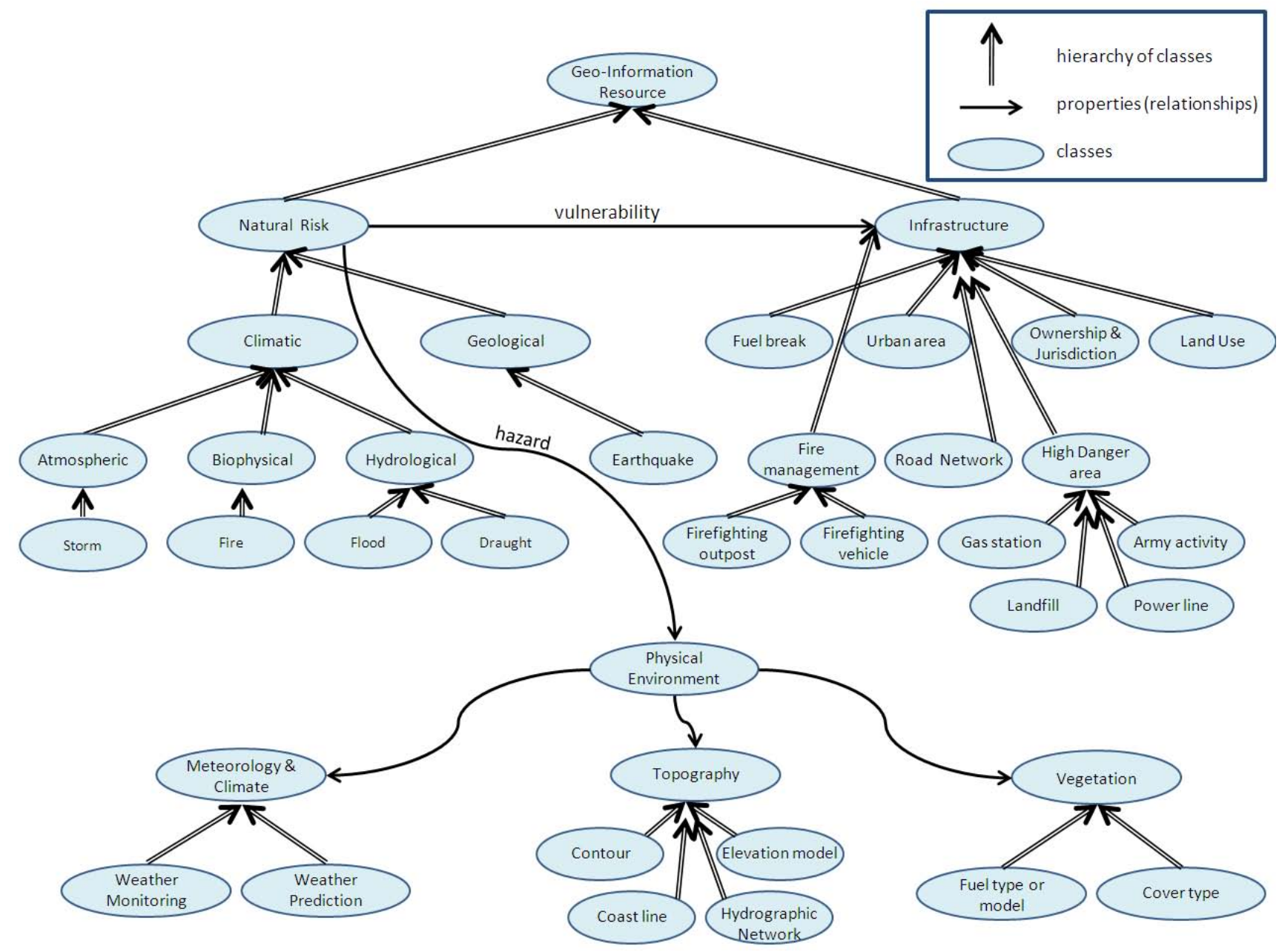

Fig. 3. The OntoFire ontology.

path by going back to the initial page and explore different data sets.

The initial page of OntoFire, represented in Fig. 5, is divided into four concrete parts. In the left part, the hierarchy of classes in the OntoFire ontology is displayed in a tree-view form in order to represent the relationships of generalization and specialization. By folding and unfolding the tree-view, users can traverse the entire hierarchy and select a specific category by clicking on its hyperlink. This action means that users would like to limit the resources found to those classified under this class or its subclasses. In the central part, an interactive map helps users to specify the bounding box of the area for searching resources of interest. In the right part, users can filter the metadata descriptions according to some basic elements of the core metadata set of ISO 19115, such as the dataset title, abstract or the reference date. The activation of the "Find" button calculates and represents resources, by taking into account:

- the class that the user selects from the tree-view form in the left part of the page;
- the bounding box of the geographic area specified from the interactive map in the central part of the page;

- the filtering conditions (if any) based on the terms of the ISO 19115 metadata elements specified in the right part of the starting page.

For every resource in the result list, the system shows the metadata title (in bold letters) and the metadata abstract. The activation of the "View Details" button shows the full metadata description of the corresponding resource (Fig. 6). This detailed description can help users to evaluate the characteristics of the resources so that they can actually check whether this information meets their requirements.

Underneath the full metadata record of the resource, the system represents hyperlinks to several classes according to the OntoFire ontology (Fig. 7). At every browsing step, users can follow the hyperlinks provided to navigate either "vertically" (to broader/narrower classes of the current one) or "horizontally" (to classes related with RDF properties such as "vulnerability" and "hazard"). Thus, if a user has already 


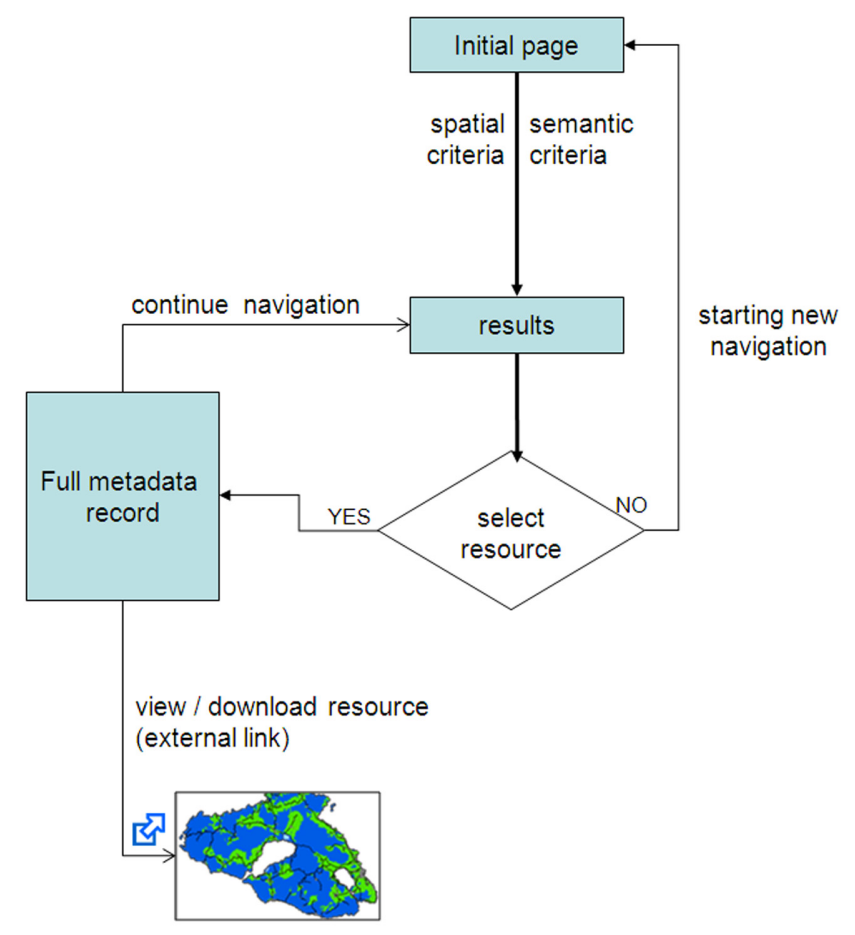

Fig. 4. Information flow in the browsing interface of OntoFire.

explored data about a specific class, OntoFire provides an easy and intuitive way to search also for data "semantically close" to the specified class in a preferred geographic area. By selecting the hyperlink of a specific class, the navigation continues by exploring resources classified under the corresponding class selected. In Fig. 7, the current category is "Fire", which is related to class "Physical Environment" through the property "hazard" and related to class "Infrastructure" through the property "vulnerability". Class "Fire" has no specific categories, thus, users cannot navigate to any more specific categories. However, they can follow the "Biophysical" hyperlink to explore resources classified under this more generic class.

Figure 8 presents graphically the semantic navigation mechanisms in OntoFire. The user has selected a resource of category Fire. As the more generic class "Natural Risk" of class "Fire" is related to classes "Infrastructure" and "Physical Environment", the system suggests the user to continue the navigation in the corresponding categories "Infrastructure" and "Physical Environment" (Fig. 8). In a similar way, it suggests the navigation to its generic category "Biophysical". The transition from the category "Fire" to class "Infrastructure" changes the set of the proposed categories and allows further exploration of related resources (Fig. 9). Thus, the field "This category" will change from "Fire" to "Infrastructure" category, since this is now the chosen category. Furthermore, the associated classes have changed and the system proposes the navigation to the category "Natural Risk", through the "vulnerability" relationship.
The semantic navigation mechanisms are seamlessly combined with spatial navigation mechanisms. Every resource carries a property called "Metadata_Geographic_Location" derived from the class "Geo-Information Resource" (see Fig. 2). This property identifies the geographic extent of each resource. Through the interactive map in the central part of OntoFire's interface (see Fig. 5), users can specify the extent of the desired area of interest. According to the extent of the interactive map and the extent of each resource, the results found at every browsing step are inside, overlap or are in a buffer zone (10,50 or $100 \mathrm{~km})$ around the current extent of the interactive map. Thus, at every browsing step, users combine the semantic navigation, by following the semantic hyperlinks, with spatial navigation according to the desired area of interest and the geographic extent of the resources.

In Fig. 10, the user explores resources of class "Physical Environment" within a specific geographic extent. Based on the underlying ontology, OntoFire suggests the user to continue her/his navigation and explore different resources in related categories such as "Meteorology_and_Climate" and "Topography". At the same time, the user has chosen to search for topography and weather data in a buffer zone of $10 \mathrm{~km}$ around an area of interest. He/she selects the corresponding classes (i.e. Meteorology_and_Climate, Topography) by activating the corresponding selection button next to each class. To find resources that satisfy these criteria, the user follows the "Find" hyperlink and results are shown in the bottom place of the page. If the user chooses a new resource from the calculated results, the graphical interface will be informed with the new metadata record and the navigation will continue in the same way.

\subsection{The publishing interface}

OntoFire allows the dynamic change of its content by providing mechanisms for publishing, modifying or removing metadata descriptions. Every resource in the geo-portal usually is classified under a specific class, depending on its corresponding semantics. For example, a map showing the fire risk for a specific area can be classified under class "Fire". Similarly, a $\mathrm{kml} / \mathrm{kmz}$ file about the positions of firefighting outposts is classified under class "Firefighting outposts", which is a specialized class of class "Fire Management". Figure 11 represents this kind of classification of resources in specific classes of the ontology. However, there is also the case where one resource to be classified under more than one class (multiple classification). For example, a resource with title "ground erosion after biannual fires in the pine forest of Lesvos" can be classified both under classes "Erosion" and "Fire" (Fig. 12).

The catalogue interface of OntoFire geo-portal allows resources to be classified under any number of classes (Fig. 13). Only the interface for submitting new metadata descriptions is shown in Fig. 13; similar interfaces exist for modifying existing metadata or removing descriptions of 


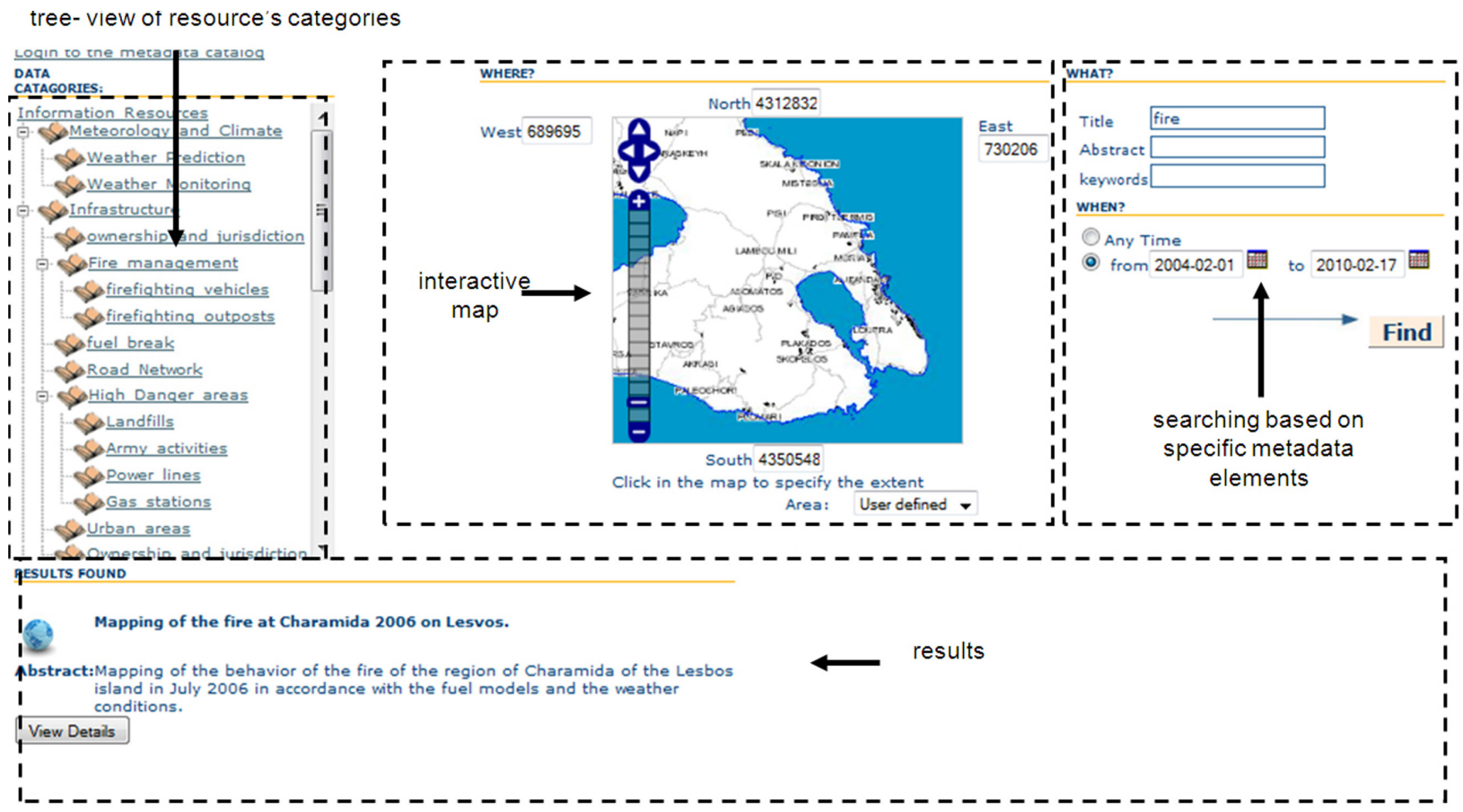

Fig. 5. The initial page in the browsing interface of OntoFire.

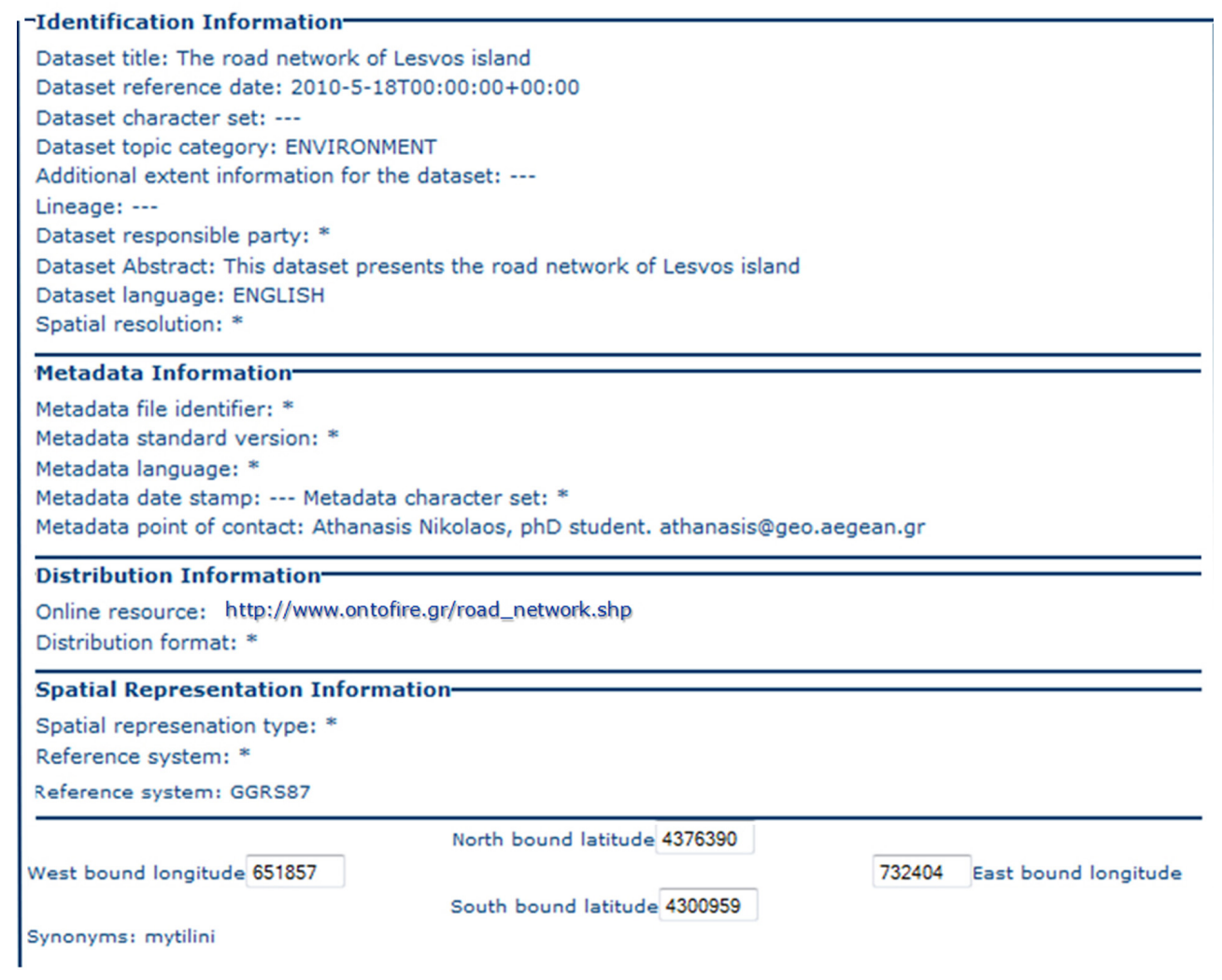

Fig. 6. Detailed metadata description of a resource in OntoFire. 
RESOURCE CATEGORIES:

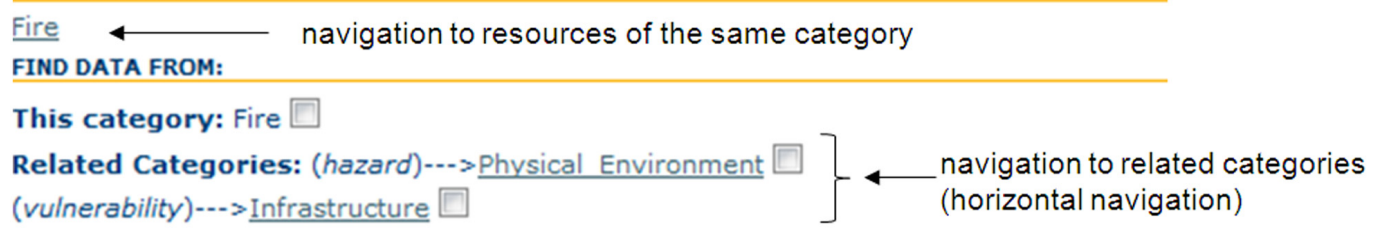

(vulnerability) ---> Infrastructure

navigation to general/specific categories

Specific categories:

General Categories: Biophysical $\square] \longleftarrow$ (vertical navigation)

Fig. 7. Semantic navigation.

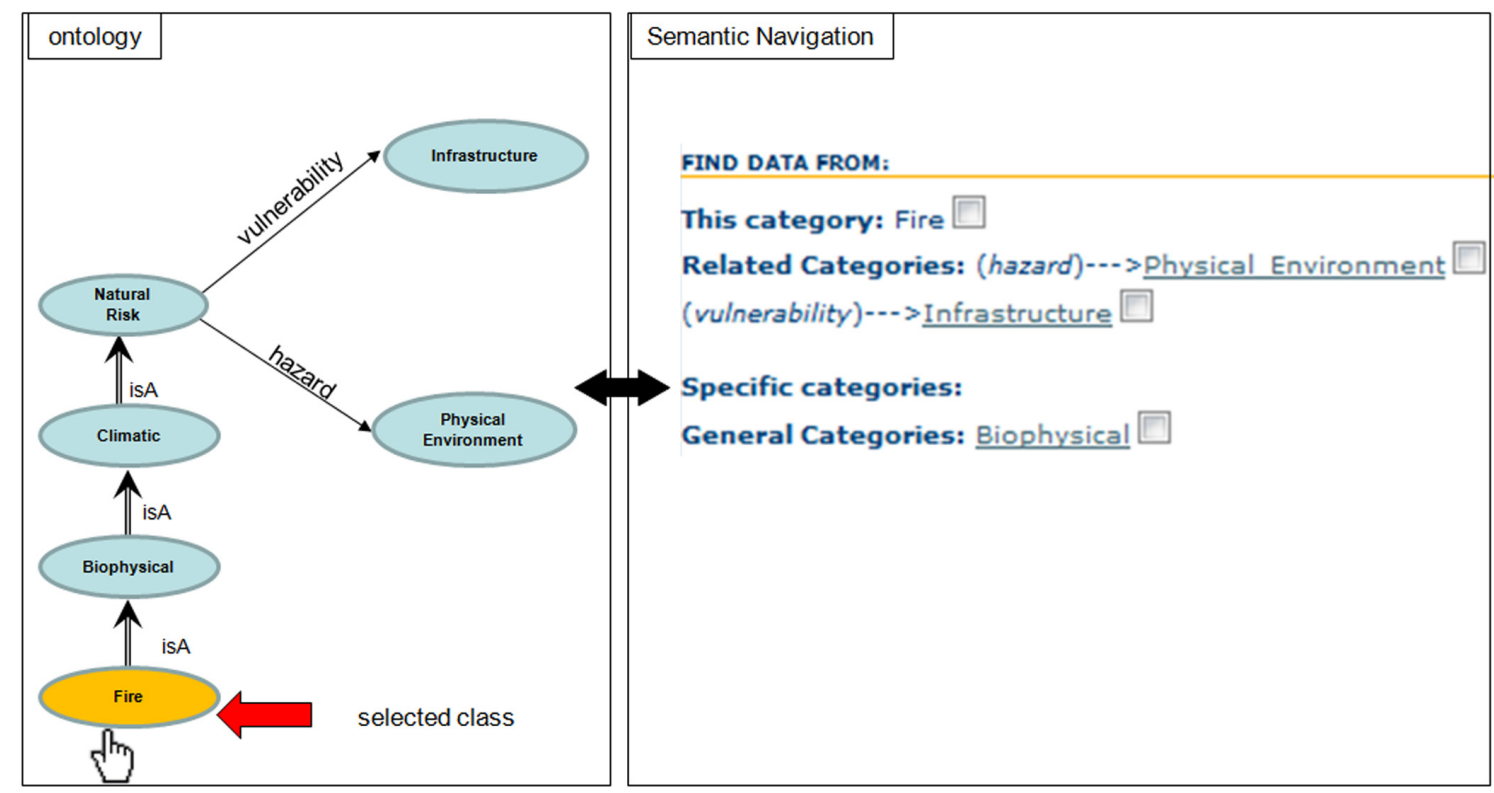

Fig. 8. Semantic navigation in OntoFire.

interest from the metadata catalogue. In the left part, the interface represents the hierarchy of the ontology's classes in a tree-view form, similar to the tree-view used for browsing purposes. Next to each class, a check box indicates that the resource will be classified under the corresponding class. By selecting more than one check box, the same resource is classified under all classes selected. In the central area, the interactive map allows users to specify the coordinates of the minimum bounding rectangle (or bounding box) of the new resource. In the right part, users can fill in the values of the metadata elements that correspond to the basic core set of ISO 19115 metadata standard. For the mandatory elements, the system shows a symbol (*) next to the corresponding field, which indicates that they have to be filled out before the provider submits the new metadata record.

\subsection{Architecture}

Figure 14 represents the three-tier architecture of OntoFire. The front end is based on the servlet container Apache
Tomcat (2011) that incorporates the open library OpenLayers for the visualization of the resources in the interactive map. At the backend, metadata descriptions are stored in a PostgreSQL (2011) object-relational database. For the semantic navigation mechanisms, the semantic query language RDF Query Language (RQL) (Karvounarakis et al., 2002) is used. RQL is part of the open source system ICS-FORTH RDFSuite (Alexaki et al., 2002) that provides: (i) semantic validation of the metadata descriptions, (ii) storage of the metadata in the PostgreSQL database, and (iii) generation of semantic queries over the semantic metadata. For the spatial navigation mechanisms, OntoFire uses PostGIS that adds support for geographic objects to the PostgreSQL database. The calculation of resources at every browsing step is based on a combination of RQL queries for the semantic part and PostGIS queries for the spatial part of it. 


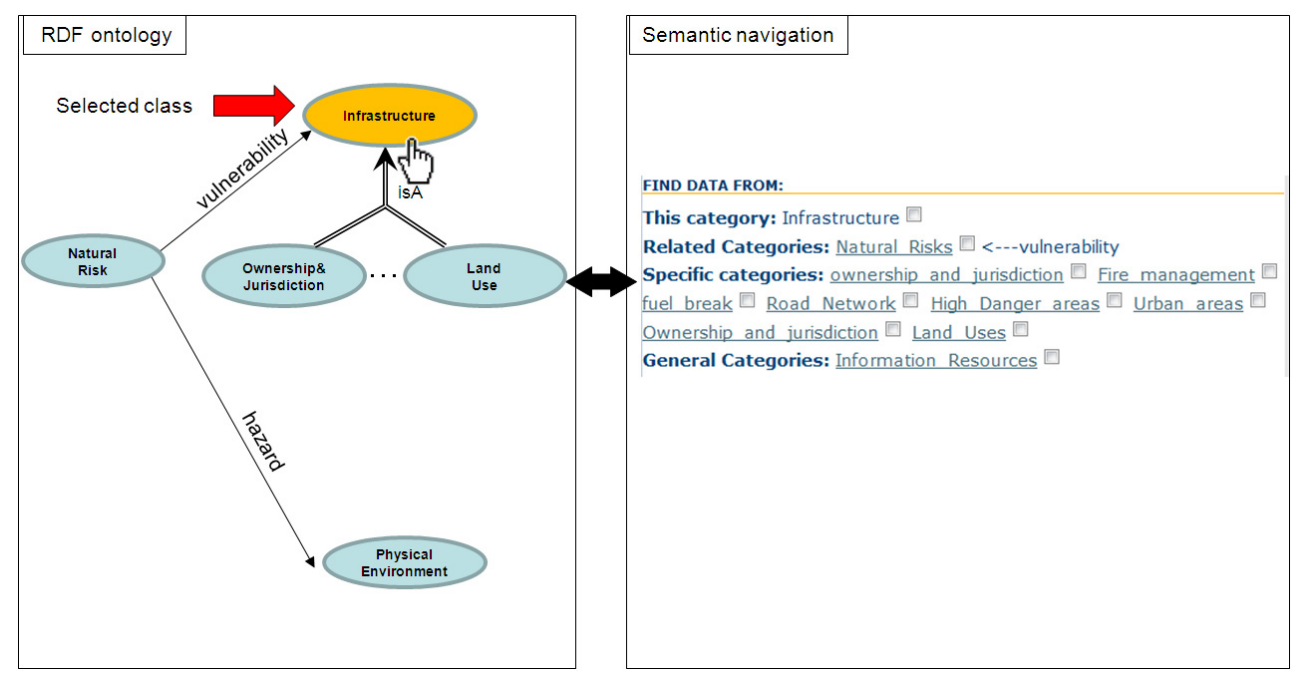

Fig. 9. Exploration of semantic related resources.

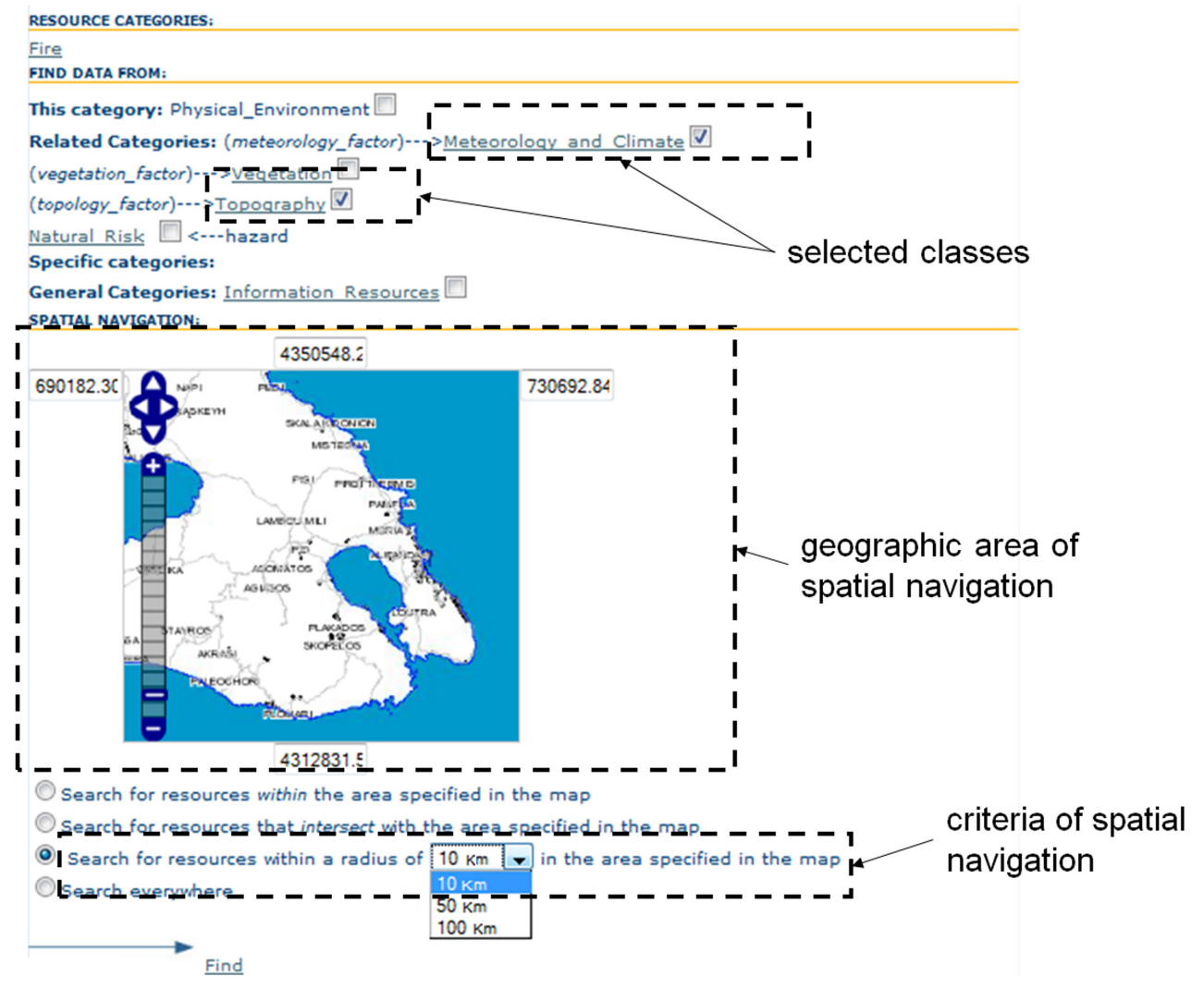

Fig. 10. Specifying the criteria of spatial navigation in OntoFire.

\section{OntoFire's evaluation}

OntoFire holds metadata descriptions about wildfire resources in the area of Lesvos Island, Aegean Sea, Greece. The system is ready to be used by local fire agencies and authorities of civil protection, as currently done during the
2011 fire season. Furthermore, the geo-portal's functionalities have been presented to 60 graduate students at the Department of Geography, University of the Aegean. The graduate students, with wide background coursework on Geo-Informatics and Environmental Risk Management, had an acquaintance with the geo-portal's innovative features 


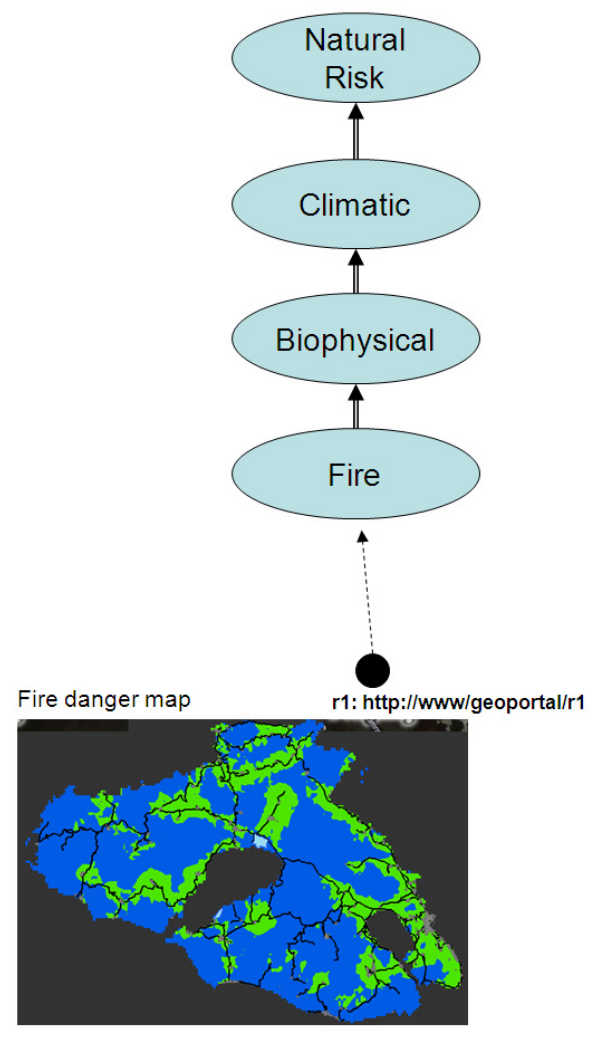

Fig. 11. Classification of resources in the OntoFire ontology.

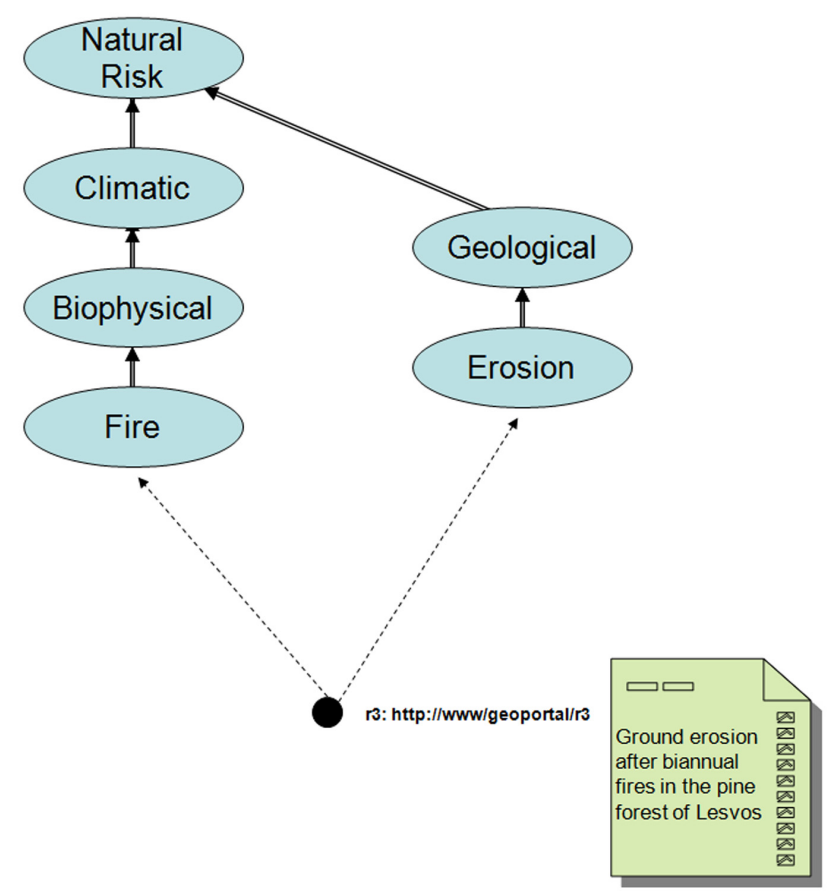

Fig. 12. Multiple classification of resources in the OntoFire ontology.

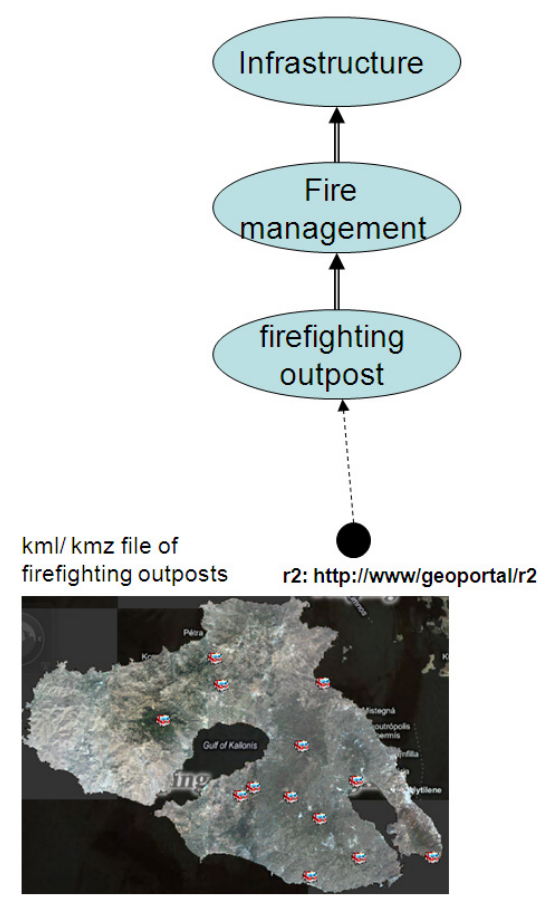

through a series of lectures. Some hypothetical scenarios were given to the students to evaluate the system's capabilities. The first scenario was to discover resources about wildfires that have already occurred in the area of Lesvos. By choosing the class "Fire" from the tree-view form, the students searched for related metadata records. They further searched for resources about the conceptually-related categories of the physical environment and the anthropogenic factors through the semantic hyperlinks of concepts "Fire", "Physical Environment" and "Infrastructure". Furthermore, they explored data about the topography, the weather and the vegetation of the burnt areas. Therefore, the students were able to derive thorough and exhaustive details about a wide range of information regarding the fires in the whole area of Lesvos Island.

The second scenario was to find useful resources about a specific fire that appeared in a specific area in the year of 2006. Many students did not even know the exact location of this area, but given the fact that it is located in the southeast part of Lesvos Island, they used the interactive map to spatially navigate to this area. The students used the interactive map to constrict the geographic area and filtered the resources found by using the year "2006" as the reference date of the fire. They explored several resources from the result's list, and derived useful information by looking into their detailed metadata description. Furthermore, by exploiting the 

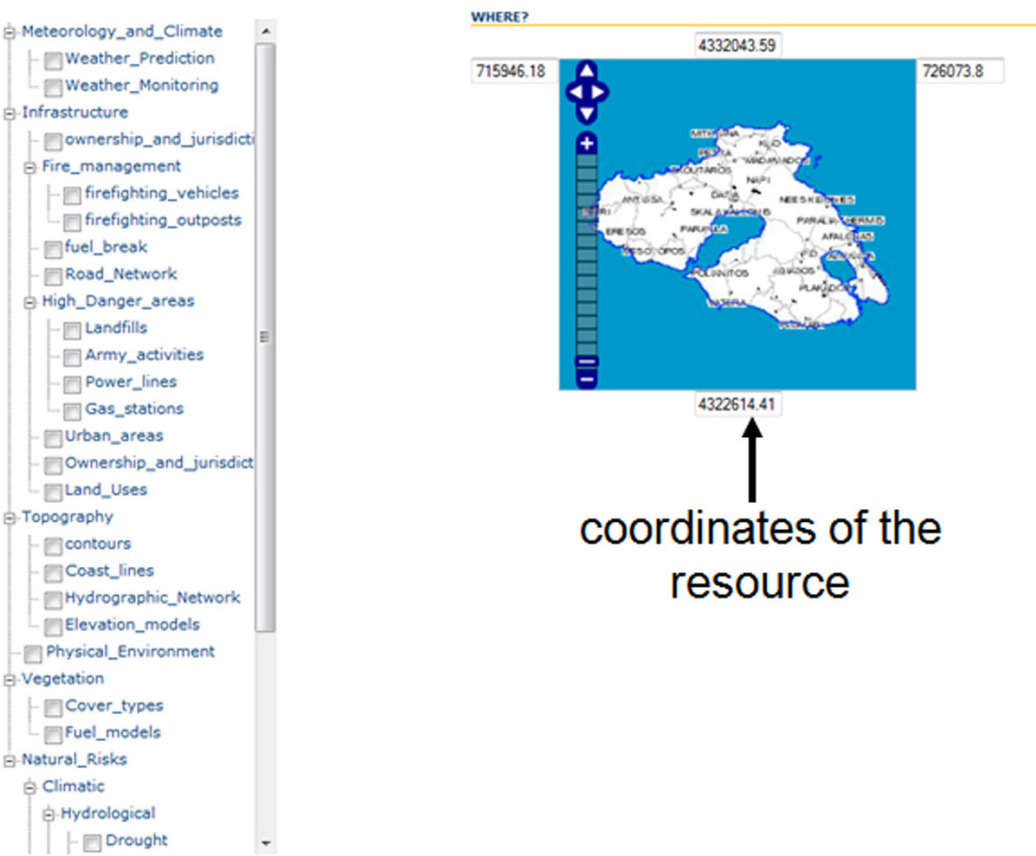

Fig. 13. Metadata publishing in OntoFire.

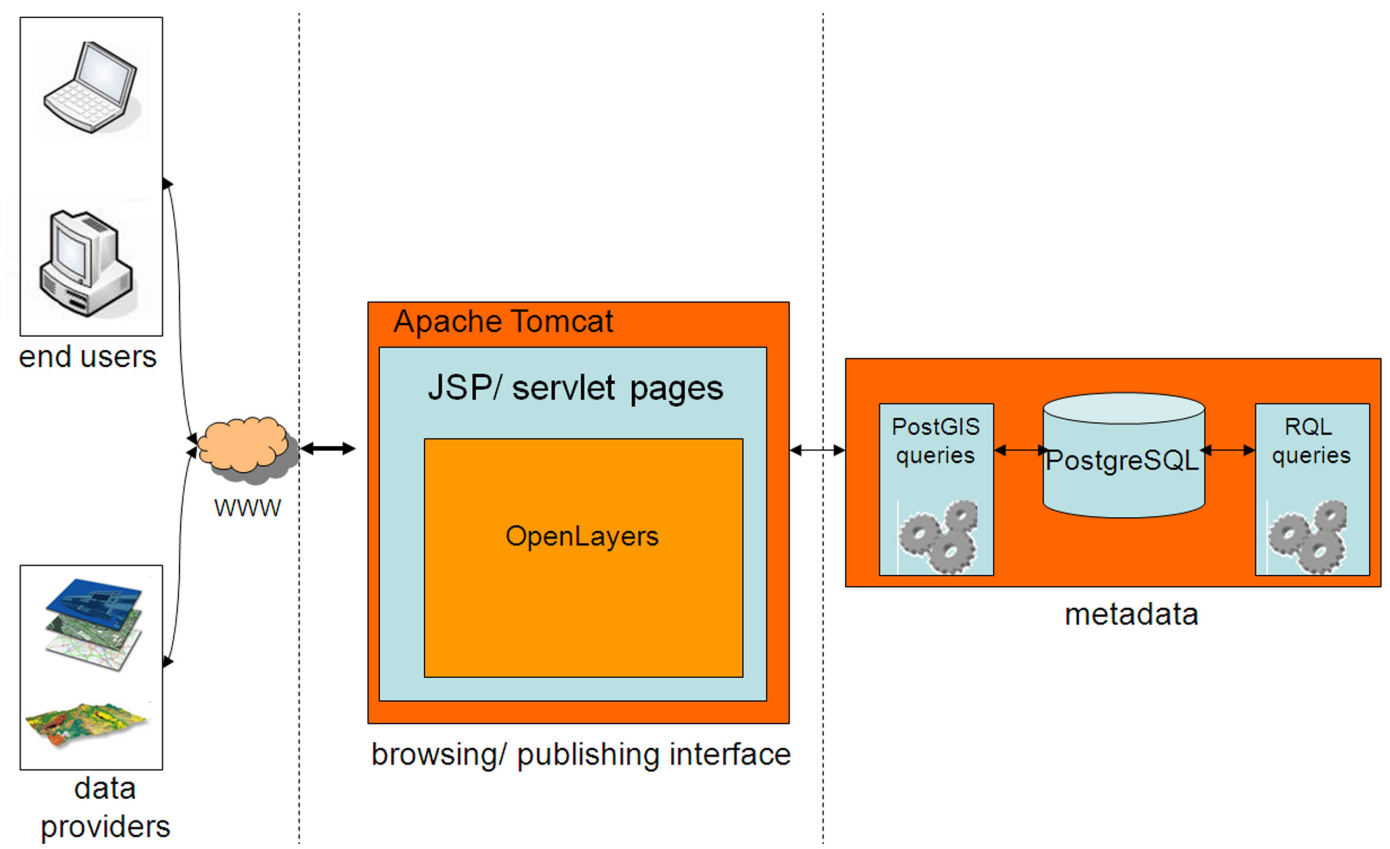

Fig. 14. The architectural design of OntoFire.
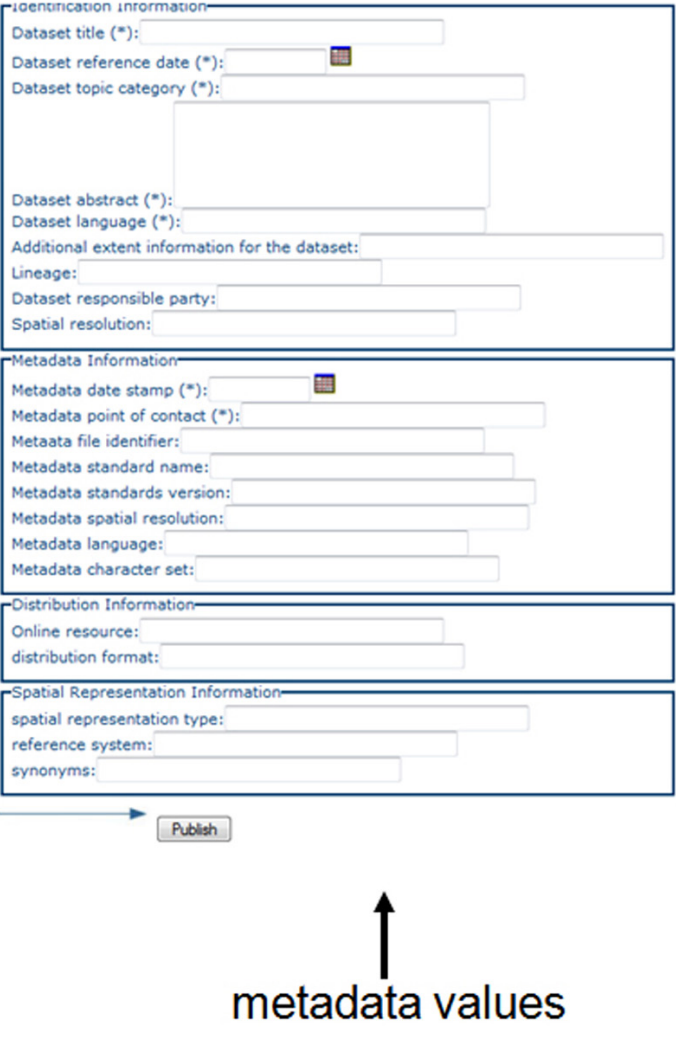
Table 1. Positive and negative impressions of OntoFire.

\begin{tabular}{ll}
\hline Positive impressions & Negative impressions \\
\hline $\begin{array}{l}\text { Easy to get familiar with the interface } \\
\text { of OntoFire }\end{array}$ & $\begin{array}{l}\text { Difficulties for people } \\
\text { that are not familiar }\end{array}$ \\
$\begin{array}{l}\text { Easy to get an efficient knowledge by } \\
\text { navigating spatially and semantically }\end{array}$ & $\begin{array}{l}\text { language } \\
\text { Effective finding of useful information } \\
\text { for prevention and planning purposes }\end{array}$ \\
\hline
\end{tabular}

semantic and spatial navigation mechanisms, they were able to:

1. Explore further related resources classified under the same class in the same geographic area, or more general resources about biophysical risks.

2. Explore further resources from the semantically related categories "Physical environment" and "Infrastructures".

3. Ask for resources included in the same area or form a buffer zone of 5,10 or $20 \mathrm{~km}$ around this area.

After completing these tasks, the students anonymously registered their comments about the system's functionality. Table 1 shows the positive and negative impressions after processing the user's feedback. This evaluation procedure revealed the contribution of OntoFire as an effective searching tool in a geo-portal environment. Users found much easier to explore resources through hyperlinks than putting keywords. They were able to complete their search much quicker and more efficiently. Analogous were the impressions from users of local fire authorities; their personnel have been trained to use the system and continued to consult it during the 2011 fire season. Internal meetings had been arranged at the fire agencies' premises to collect all their experiences from using the system in order to validate the application's functionality. By finding several data validly and promptly, local fire authorities were able to extract useful information towards the design of an effective operational wildfire prevention and management plan.

\section{Conclusions}

Geo-portals play the role of SDI gateways by providing single access points to multiple emergency data and facility resources. In the area of wildfires, geoportals can provide valuable help for user communities about risk knowledge, prevention strategies and information dissemination. We have presented OntoFire, an ontology-based geo-portal about wildfires. Unlike conventional geo-portals that rely on keywords-typing for searching data of interest, OntoFire leverages the semantic and spatial relationships between the resources by providing ontology-based and spatially-based navigation mechanisms. To the best of our knowledge, OntoFire is the first geo-portal specialized in the area of wildfires which uses a corresponding ontology to enrich the data in a semantically meaningful way. The coexistence of the semantic and the spatial navigation mechanisms gives users the ability to find readily what they are looking for. The proposed approach can improve the discovery of valuable information that is necessary to set priorities for disaster mitigation and prevention strategies. OntoFire aspires to be a focal point of integration and management for a very large amount of information, contributing in this way to the dissemination of knowledge and to the preparedness of the operational stakeholders in natural disasters.

Based on the OntoFire ontology, end users exploit the proposed innovative semantic and spatial navigation mechanisms to find data of interest, while data providers also afford the privilege to enrich the system with new semantically annotated metadata descriptions. End users can use the browsing interface of OntoFire to find resources of interest by using the semantic and spatial navigation mechanisms provided. Furthermore, data providers can use the publishing interface to submit new metadata, modify existing metadata or even remove descriptions of interest from its metadata catalogue. The browsing interface is open to everyone, while the publishing interface can be used only by authorized users.

Currently, OntoFire holds metadata descriptions about wildfire resources in the area of Lesvos Island, Greece. As a next step, we plan to broaden the area of coverage by including data from a more extensive region of Greece. Both the graphical interface for browsing and the graphical interface for managing the metadata catalogue will be translated into Greek in order to be operational for people not familiar with the English language.

A future research goal is to use a semantic language with higher expressiveness to further enrich the OntoFire ontology. The ontology Web Language (OWL) (McGuinness and van Harmelen, 2004) is currently used as the main language for the development of ontologies on the Internet. It is a more sophisticated model of ontological representation compared with that of the RDF Schema. It has a much wider expressiveness and uses reasoning to conduct conclusions. The exploitation of ontologies expressed in the OWL language would facilitate the development of semantic geo-portals for different scopes by using ontologies expressed in OWL that have been developed for the specific domain.

Acknowledgements. Work for this paper was partially supported through a Microsoft Research grant to the Geography of Natural Disasters Laboratory, Department of Geography, University of the Aegean, Greece. Thanks are also due to referees for their helpful reviews.

Edited by: K.-T. Chang

Reviewed by: D. Bran and another anonymous referee 


\section{References}

Apache Tomcat: available at: http://tomcat.apache.org/, last access: 1 August 2011.

Athanasis, N., Kalabokidis, K., Vaitis, M., and Soulakellis, N.: The emerge of semantic geoportals, Lect. Notes Comput. Sci., 3762, 1127-1136, 2005.

Athanasis, N., Kalabokidis, K., Vaitis, M., and Soulakellis, N.: Towards a semantics-based approach in the development of geographic portals, Comput. Geosci., 35, 301-308, doi:10.1016/j.cageo.2008.01.014, 2009.

Alexaki, S., Athanasis, N., Christophides, V., Karvounarakis, G., Magkanaraki, A., Plexousakis, D., and Tolle, K.: The ICSFORTH RDFSuite: High-level Scalable Tools for the Semantic Web, ERCIM News, No. 51, 2002.

Annoni, A., Bernard, L., Douglas, J., Greenwood, J., Laiz, I., Lloyd, M., Sabeur, Z., Sassen, A., M., Serrano, J., and Uslaender, T.: Orchestra: Developing a unified open architecture for risk management applications, 1-17, 2005.

Babitski, G., Bergweiler, S., Grebner, O., Oberle, D., and Paulheim, H.: SoKNOS - Using Semantic Technologies in Disaster Management Software, Lect. Notes Comput. Sci., 6644, 183-197, 2011.

Bernard, L., Einspanier, U., Lutz, M., and Portele, C.: Interoperability in GI Service Chains The Way Forward, in: 6th AGILE Conference on Geographic Information Science, edited by: Gould, M., Laurini, R., and Coulondre, S., 179-188, 2003.

Berners-Lee, T., Hendler, J., and Lassila, O.: The Semantic Web, Scientific American, 184, 34-43, 2001.

Blaikie, P., Cannon, T., Davis, I., and Wisner, B.: At Risk: Natural Hazards, People's Vulnerability and Disasters, Routledge, London, 1994.

Brickley, D. and Guha, R. V.: RDF Vocabulary Description Language 1.0: RDF Schema, W3C Recommendation, 2004.

Cannon, T.: Vulnerability Analysis and the Explanation of "Natural" Disasters, in: Disasters, Development and Environment, edited by: Varley, A., Chichester, New York, Brisbane, Toronto and Singapore, John Wiley \& Sons, 13-29, 1994.

Chen, K., Blong, R., and Jacobson, C.: Towards an integrated approach to natural hazards risk assessment using GIS: with reference to bushfires, J Environ. Manage., 31, 546-560, 2003.

Christensen, F., Bernard, A., Kanellopoulos, L., Nogueras, I., Schade, J. I., and Thorne, C: Building service oriented applications on top of a spatial data infrastructure - a forest fire assessment example, Proceedings of the 9th International AGILE Conference, 2006.

Drafting Team Metadata and European Commission Joint Research Centre: INSPIRE Metadata Implementing Rules: Technical Guidelines based on EN ISO 19115 and EN ISO 19119, MD_IR_and_ISO_20090218, 2009.

ESRI ArcGIS for Server: available at: http://www.esri.com/ software/arcgis/arcgisserver/index.html, last access: $1 \mathrm{Au}-$ gust 2011.

Fu, G., Jones, C. B., and Abdelmoty, I.: Ontology-Based spatial query expansion in information retrieval, Volume 3761/2005, Springer Berlin/Heidelberg, 2005.

Galindo, P. A.: Integration of Forest Fire Management with SDI: User requirements, MSc in Geospatial Technologies, 2010.

GeoServer web site: available at: http://geoserver.org/display/ GEOS/Welcome, last access: 1 August 2011.
Giuliani, G. and Peduzzi, P.: The PREVIEW Global Risk Data Platform: a geoportal to serve and share global data on risk to natural hazards, 53-66, 2011.

Google Maps API: available at: http://code.google.com/intl/el-GR/ apis/maps/, last access: 1 August 2011.

Grasso, F.: Early Warning Systems: State-of-Art Analysis and Future Directions, UNEP Draft Report, 2009.

Gwenzi, J.: Enhancing Spatial web search with Semantic Web Technology and Metadata Visualisation, GeoInformation Science, 2010.

Hochmair, H.: Ontology matching for spatial data retrieval from Internet portals, in: Proceedings of Geospatial Semantics, Lect. Notes Comput. Sci., Mexico City, Mexico, 3799, 166-182, 2005.

Ilyas, Q. M. and Ahmad, I.: A Conceptual Architecture of SAHARA,- A Semantic Disaster Management System, World Appl. Sci. J, ISSN 1818-4952, IDOSI Publications, 10, 980-985, 2010.

Joshi, H., Seker, R., Bayrak, C., Ramaswamy, S., and Connelly, J. B.: Ontology for disaster mitigation and planning, in: Proceedings of the 2007 summer computer simulation conference (SCSC), Society for Computer Simulation International, San Diego, CA, USA, article 26, 8 pp., 2007.

Kalabokidis, K. D., Gatzojannis, S., and Galatsidas, S.: Introducing wildfire into forest management planning: towards a conceptual approach, Forest Ecol. Manage., 158, 41-50, 2002.

Karvounarakis, G., Alexaki, S., Christophides, V., Plexousakis, D., and Scholl, M.: A Declarative Query Language for RDF, The Eleventh International, World Wide Web Conference (WWW'02), 2002.

Klien, E., Lutz, M., and Kuhn, W.: Ontology-based discovery of geographic information services. An application in disaster management, Comput. Environ. Urb. Sys., 30, 102-123, 2006.

Köhler, P. and Wächter, J.: Towards an open information infrastructure for disaster research and management: Data management and information systems inside DFNK, Nat. Hazards, 38, 141157, 2006.

Köhler, P., Müller, M., Sanders, M., and Wchter, J.: Data management and GIS in the Center for Disaster Management and Risk Reduction Technology (CEDIM): from integrated spatial data to the mapping of risk, Nat. Hazards Earth Syst. Sci., 6, 621-628, doi:10.5194/nhess-6-621-2006, 2006.

Kolas, D.: Supporting Spatial Semantics with SPARQL, Transactions in GIS, 12, 5-18, doi:10.1111/j.1467-9671.2008.01132.x, 2008.

Lan, G. W., Zhou, X. Q., and Huangc, Y.: Integration of geographic information services for disaster management The International Archives of the Photogrammetry, Remote Sensing and Spatial Information Sciences, Vol. XXXVII, Part B4, Beijing, 2008.

Lassila, O. and Swick, R.: Resource Description Framework (RDF) Model and Syntax Specification, World Wide Web Consortium, Recommendation REC-rdf-syntax-19990222, 1999.

Lee, Y. C. and Chan, H. C. E.: Spatial metadata and its management, in: Proceedings of the International Symposium on Spatial Data Quality, Geomatica, 54, 451-462, 2000.

Lucarella, D. and Zanzi, A.: A visual retrieval environment for hypermedia information systems, ACM Trans. Info. Sys., 14, 3-29, 1996.

Lutz, M.: Ontology-Based Descriptions for Semantic Discovery and Composition of Geoprocessing Services, Geoinformatica, 
11, 1-36, 2006.

Lutz, M. and Klien, E.: Ontology-based retrieval of geographic information, Int. J. Geogr. Inf. Sci., 20, 233-260, 2006.

MapServer Open Source Web Mapping: available at: http:// mapserver.org/, last access: 1 August 2011.

Marshall, C. C., and Shipman, F. M.: Spatial hypertext and the practice of information triage, in: Proceedings of the eighth ACM conference on Hypertext (HYPERTEXT '97), edited by: Bernstein, M., Osterbye, K., and Carr, L., ACM, New York, NY, USA, 124-133, doi:10.1145/267437.267451, 1997.

McGuinness, D. L. and van Harmelen, F.: OWL Web Ontology Language Overview.W3C Recommendation, 2004.

Microsoft Bing Maps: available at: http://www.microsoft.com/ maps/developers/web.aspx, last access: 1 August 2011.

Murgante, B., Scardaccione G., and Las Casas, G.: Building ontologies for disaster management: seismic risk domain, in: Krek, A., Rumor, M., Zlatanova, S., and Fendel, E. M., Urban and Regional Data Management, CRC Press, Taylor \& Francis, London 259-269, ISBN: 978-0-415-055642-2, 2009.

Paulheim, H. and Meyer, L.: Ontology-based Information Visualization in Integrated UIs, in: Proceedings of the 2011 International Conference on Intelligent User Interfaces (IUI), ACM, New York, 451-452, 2011.

Pausas, J. G., Llovet, J., Rodrigo, A., and Vallejo, R.: Are wildfires a disaster in the Mediterranean basin? - A review, Int. J. Wildland Fire, 17, 713-723, 2008.

PostGIS: available at: http://postgis.refractions.net/, last access: 1 August 2011.

PostgreSQL: available at: http://www.postgresql.org/, last access: 1 August 2011.
Pyne, S. J., Andrews, P. L., and Laven, R. D.: Introduction to Wildland Fire 2nd Edn., Wiley \& Sons, NY, 1996.

Robichaud, P. R., Lewis, S. A., Brown, R. E., and Ashmun, L. E.: Emergency post-fire rehabilitation treatment effects on burned area ecology and long-term restoration, Fire Ecol. Special Issue, 5, 115-128, 2009.

Schroeder, M. J., and Buck, C. J.: Fire weather, US Dep. Agric., Agric. Handb., 360, 229 pp., 1970.

Shvaiko, P., Ivanyukovich, A., Vaccari, L., Maltese, V., and Farazi, F.: A semantic geo-catalogue implementation for a regional SDI, in: Proceedings of the INSPIRE conference, 2010.

Silva, A. T. and Madurapperuma, A., P.: Ontology Driven Approach to Disaster Management. Sri Lanka Association for Artificial Intelligence (SLAAI), Proceedings of the fourth Annual Sessions, Colombo, 2007.

Stocks, B., Fromm, M., Goldammer, J., Carr, R., and Sukhinin, A.: Extreme Forest Fire Activity in Western Russia in 2010: Fire Danger Conditions, Fire Behavior and Smoke Transport, Geophys. Res. Abstr., 13, EGU2011-5063, 2011 EGU General Assembly 2011, 2011.

Vasilakos, C., Kalabokidis, K., Hatzopoulos, J., and Matsinos, I.: Identifying wildland fire ignition factors through sensitivity analysis of a neural network, Nat. Hazards, 50, 125-143, 2009.

Zaharia, M. H., Leon, F., and Atanasiu, G. M.: Increasing Awareness to Disasters by Knowledge Management with Intelligent Agents, Proceedings of the 10th European Conference on Knowledge Management, Volume One, edited by: Bolisani, E. and Scarso, E., Academic Publishing Limited, Reading, UK, ISBN 978-1-906638-40-5, 906-914, 2009

Zhai, X., Huang, L., and Xiao, Z.: Geo-spatial query based on extended SPARQL, in: Proceedings of Geoinformatics, 1-4, 2010. 